\title{
La dimension internationale : un enjeu éducatif
}

\section{Hugh Starkey}

\section{OpenEdition}

Journals

Édition électronique

URL : http://journals.openedition.org/ries/4062

DOI : 10.4000/ries.4062

ISSN : 2261-4265

\section{Éditeur}

Centre international d'études pédagogiques

\section{Édition imprimée}

Date de publication : 1 juin 1995

Pagination : 47-53

ISSN : 1254-4590

\section{Référence électronique}

Hugh Starkey, "La dimension internationale : un enjeu éducatif », Revue internationale d'éducation de Sèvres [En ligne], 06 | 1995, mis en ligne le 01 janvier 2015, consulté le 01 mai 2019. URL : http:// journals.openedition.org/ries/4062 ; DOI : 10.4000/ries.4062

Ce document a été généré automatiquement le 1 mai 2019.

(c) Tous droits réservés 


\title{
La dimension internationale : un enjeu éducatif
}

\author{
Hugh Starkey
}

1 Les systèmes nationaux de l'enseignement en Europe ne peuvent plus s'abstenir de confronter la réalité d'un monde de nations de plus en plus interdépendantes. L'évolution de l'Union européenne touche tous les habitants de l'Europe et ne peut être exclue ni du programme scolaire ni de la formation initiale des enseignants. Un financement assez important est déjà en place pour développer une dimension européenne de l'enseignement et cet effort sera renforcé par le programme Socrate et ses composants Erasmus pour les universités et Comenius pour les écoles. Mais l'interdépendance des nations n'est pas limitée à l'Europe. Les difficiles négociations du GATT sont la preuve d'une économie mondiale profondément imbriquée.

Une dimension internationale de la formation initiale des enseignants est indispensable pour des raisons liées au développement économique de la planète et au besoin de former de futurs citoyens capables de trouver leur place dans ce monde. Bien que les impératifs économiques semblent dominer l'évolution de la société, les questions sociales et éthiques sont globalement importantes. La stabilité de nos sociétés n'est pas assurée. Elle ne va pas de soi. Les conflits interethniques meurtriers sévissent au sein même de l'Europe. Les futurs enseignants ont un rôle primordial qui consiste à aider les jeunes à vivre en harmonie et à favoriser une évolution sociale basée sur « la liberté, la justice et la paix dans le monde » selon la formule de la Déclaration universelle des droits de l'homme. Il est impossible de construire la paix dans le monde ou même à l'intérieur d'une société multiculturelle sans avoir connaissance des grands principes éthiques communs adoptés par les nations. Ce n'est pas un hasard si les chefs d'États signataires du traité d'Union européenne, dit traité de Maastricht, tiennent à confirmer au début du préambule, et avant d'aborder la dimension économique, « leur attachement aux principes de la liberté de la démocratie, du respect des droits de l'homme, des libertés fondamentales et de l'état de droit ». 


\section{État d'urgence}

3 «Urgences » est le titre d'une section du Rapport de l'Inspection générale de l'éducation nationale $1994^{1}$ qui traite de l'enseignement de l'histoire, de la géographie et de l'éducation civique. La première de ces urgences est que « le monde a changé ».

Il s'agit de prendre acte de ce qui advient depuis 1989 [...] quand se conjuguent des nouveautés aussi fortes que l'implosion du communisme et la fin de l'Empire soviétique, la crise mondiale multiforme, le recul des idéologies linéaires, les mutations démographiques, l'explosion urbaine, la déconstruction des ruralités, l'avènement de sociétés développées à deux vitesses, les démultiplications technologiques de la connaissance et de la communication, les décalages si sensibles entre le politique, le social et le culturel, etc. Sur toutes ces questions en suspens, le système éducatif ne peut pas rester muet. Car des élèves et leurs parents le souhaitent, des enseignants ont depuis longtemps pris la parole et toutes les interdictions sont tournées (sur l'évocation en classe de la guerre du Golfe en 1991, par exemple) : une demande sociale existe et l'école a pour mission de ne pas la décevoir, tout en la reformulant au besoin.

4 Ces changements dans la situation mondiale n'ont pas toujours leur équivalent dans les mentalités. Les structures de l'éducation restent, par certains égards, ancrées dans des traditions du xIX siècle. En France, à cette époque, la dimension nationale de l'éducation avait pour mission d'élargir l'horizon des élèves jusqu'alors souvent limité aux environs de la commune. Il fallait dépasser les particularismes régionaux (langue et traditions, par exemple) pour créer un sentiment d'appartenance à un ensemble national. Selon Christian Nique $^{2}$, la réforme des programmes de l'École normale publiée le 3 août 1881 marque un changement profond dans l'orientation de l'éducation nationale en France. Jusqu'alors, «la doctrine qui prévalait était que pour assurer l'ordre social il fallait par l'école former de bons chrétiens. Avec le retour en force de la République, l'instruction religieuse est supprimée. En revanche, la première des matières d'enseignement aux élèves-maîtres est l'instruction morale et civique, et il est précisé qu'elle sera enseignée non par des maitres-adjoints ordinaires, mais par le directeur lui-même ».

5 À la fin du siècle dernier, l'éducation civique, qui était une éducation à la dimension nationale, avait la place la plus prestigieuse dans la formation des enseignants et, selon Jules Ferry, dans le programme de l'école :

En vous dispensant de l'enseignement religieux, on n'a pas songé à vous dispenser de l'enseignement moral: c'eût été vous enlever ce qui fait la dignité de votre profession. Au contraire, il a paru tout naturel que l'instituteur, en même temps qu'il apprend aux enfants à lire et à écrire, leur enseigne aussi ces règles élémentaires de la vie morale qui ne sont pas moins universellement acceptées que celles du langage ou du calcul's.

6 Mais cette dimension nationale, qui à l'époque ouvrait les esprits, ne correspond plus, à la veille $\mathrm{du} \mathrm{xxI}^{\mathrm{e}}$ siècle, aux horizons politique, économique et social des gouvernements. Pourtant l'éducation civique, dans sa conception française, repose sur un attachement à des valeurs universelles, ces valeurs "universellement acceptées». Les citoyens, pour leur part, rattachés depuis un moment au câble et au satellite et recevant des images et des informations de partout sur la planète, peuvent douter de l'universalité de ces valeurs. Le moment est donc venu d'affirmer et de démontrer cette universalité. En France, comme dans plusieurs autres pays, l'éducation civique est inscrite au programme de l'école et donc aussi au programme de formation des enseignants. Elle n'est plus au 
premier rang des matières et son prestige d'autrefois s'est effrité. Face à une population scolaire de plus en plus hétéroclite, et venant d'horizons et de milieux très divers, les enseignants auraient-ils abandonné la transmission de valeurs? N'y-a-t-il plus besoin d'assurer l'ordre ? Le nouveau catéchisme laïc serait-il l'affaire de la télévision? Ou bien serait-il une affaire familiale privée ? N'y aurait-il plus de valeurs communes?

\section{Des valeurs communes}

7 En effet, les chefs d'États des trente-deux pays du Conseil de l'Europe réunis à Vienne, en octobre 1993, ont déclaré :

Tous nos pays sont attachés à la démocratie pluraliste et parlementaire, à l'indivisibilité et à l'universalité des droits de l'homme, à la prééminence du droit, à un patrimoine culturel enrichi de ses diversités. Ainsi, l'Europe peut devenir un vaste espace de sécurité démocratique. Cette Europe est porteuse d'un immense espoir qui, à aucun prix, ne doit être détruit par des ambitions territoriales, la renaissance de nationalismes agressifs, la perpétuation des zones d'influence, l'intolérance ou les idéologies totalitaires.

8 Le premier de ces paragraphes affirme l'existence de valeurs communes aux États européens. Le second contient un programme éducatif, car l'éducation est par définition portée vers l'avenir et elle est donc source d'espoir. Il incombe à l'éducation de promouvoir l'espoir et combattre les idéologies et les mouvements qui le menacent. On peut même dire que c'est en promouvant l'espoir, qui vient d'un attachement aux valeurs des droits de la personne ${ }^{4}$, que les enseignants peuvent au mieux combattre ces menaces. Les idéologies racistes et xénophobes ne sont-elles pas issues d'un pessimisme profond quant à la situation économique, sociale et politique? Ne véhiculent-elles pas un pessimisme en ce qui concerne la nature humaine? Une éthique des droits de la personne, par contre, est optimiste car elle cherche la construction de «la justice et la paix dans le monde ».

9 Si les États européens peuvent affirmer leur adhésion aux valeurs communes que sont la liberté, la démocratie, le respect des droits de l'homme et des libertés fondamentales et l'état de droit, qu'en est-il au niveau mondial ? Le 25 juin 1993, la Conférence mondiale des droits de l'homme a adopté, à l'unanimité des 171 États représentés (dans lesquels vivent $99 \%$ de la population de la planète), une déclaration et un programme d'action. L'article premier de la déclaration affirme :

La Conférence mondiale des droits de l'homme réaffirme l'engagement solennel de tous les États de remplir leurs obligations à promouvoir le respect universel, la protection de tous les droits de l'homme et les libertés fondamentales pour tous en accord avec la Charte des Nations Unies, les autres instruments relatifs aux droits de l'homme et le droit international. L'universalité de ces droits et ces libertés n'est pas en question.

10 Cette confirmation de l'universalité des droits de la personne est suivie dans les articles 78-82 par une affirmation de l'importance d'une éducation aux droits de la personne :

L'éducation aux droits de la personne doit comprendre la paix, la démocratie, le développement et la justice sociale, tels qu'ils sont les instruments internationaux et régionaux, pour arriver à un entendement commun et une sensibilisation afin de renforcer les engagements universels visant le respect des droits de la personne ${ }^{5}$. 


\section{Pour une dimension internationale de l'éducation}

11 Il y a une multiplicité de déclarations, de résolutions et de recommandations des instances européennes et mondiales visant à promouvoir une dimension internationale et interculturelle de l'éducation. Je citerai principalement celles de l'UNICEF et de l'UNESCO. Pour Bronislaw Geremek, le Conseil de l'Europe est « l'institution européenne la mieux à même de fournir le contenu spirituel de l'idée européenne dans son expression la plus simple et la plus naturelle : la philosophie des droits de l'homme ${ }^{6} »$. Les 34 États membres du Conseil de l'Europe adhèrent tous à la Convention européenne de sauvegarde des droits de l'homme et des libertés fondamentales. Selon le préambule de la Convention, les libertés fondamentales, sans lesquelles la justice et la paix dans le monde ne sont pas assurées, reposent sur « un régime politique véritablement démocratique, d'une part et d'autre part, sur une conception commune et un commun respect des droits de l'homme dont ils se réclament ». Les Européens ont-ils « une conception commune» des droits de la personne? On peut s'interroger étant donné le peu d'écho donné aux droits de la personne dans certains pays, y compris l'Angleterre où, en 1994, le gouvernement a rayé la seule mention de la Déclaration universelle du programme d'histoire du collège. Si le public est ignorant des droits et des instruments internationaux, est-ce que ces droits vont continuer à être protégés ? Les pouvoirs publics semblent en douter, et en 1985, le comité des ministres de l'éducation du Conseil de l'Europe a fait une recommandation «sur l'enseignement et l'apprentissage des droits de l'homme dans les écoles ». Le comité des ministres signataires de la recommandation était «conscient de la nécessité de réaffirmer les valeurs de la démocratie face à l'intolérance, aux actes de violence et au terrorisme, à la résurgence d'attitudes racistes et xénophobes qui s'expriment ouvertement, au désenchantement de nombreux jeunes Européens, touchés par la récession économique et conscients de la persistance de la pauvreté et l'inégalité dans le monde»; en conséquence les ministres estiment que "tout au long de leur cursus scolaire, tous les jeunes devraient se familiariser avec les droits de l'homme dans le cadre de leur préparation à la vie dans une démocratie pluraliste ».

12 La recommandation contient en annexe des suggestions détaillées concernant le contenu d'un tel enseignement et les conditions dans lesquelles il est dispensé au mieux. Il y a aussi une section sur la formation des enseignants :

La formation initiale des enseignants devrait les préparer au rôle qu'ils seront appelés à jouer dans l'enseignement des droits de l'homme. Les futurs enseignants devraient, par exemple, être incités à s'intéresser aux affaires intérieures et internationales, avoir l'occasion d'étudier ou de travailler à l'étranger ou dans un milieu différent; apprendre à discerner et à combattre toutes les formes de discrimination dans les écoles et la société, et être encouragés à affronter et à vaincre leurs propres préjugés.

Une éducation aux droits de la personne est donc nécessairement une éducation ayant une importante dimension internationale. On peut dire aussi qu'une éducation ayant une dimension internationale a besoin d'une dimension éthique qui est celle fournie par les droits de la personne.

Les États européens font partie aussi des instances mondiales comme l'UNICEF et l'UNESCO. Dans le cadre de l'UNICEF les États ont été invités, en 1989, à signer la Convention des droits de l'enfant. Jamais un instrument de droit international n'a connu un si grand succès en si peu de temps. En deux ans, plus d'une centaine de pays y ont 
adhéré et il y en a très peu actuellement qui résistent. Cette Convention, contraignante pour les États, contient deux articles concernant l'éducation, dont l'article 29 traite des finalités :

- «favoriser l'épanouissement de la personnalité de l'enfant et le développement de ses dons et de ses aptitudes mentales et physiques, dans toute la mesure de leurs potentialités ;

- inculquer à l'enfant le respect des droits de l'homme et des libertés fondamentales, et les principes consacrés dans la Charte des Nations unies;

- inculquer à l'enfant le respect de ses parents, de son identité, de sa langue et de ses valeurs culturelles, ainsi que le respect des valeurs nationales du pays dans lequel il vit, du pays duquel il peut être originaire et des civilisations différentes de la sienne ;

- préparer l'enfant à assumer les responsabilités de la vie dans une société libre, dans un esprit de compréhension, de paix, de tolérance, d'égalité entre les sexes et d'amitié entre tous les peuples et groupes ethniques, nationaux et religieux, et avec les personnes d'origine autochtone ;

- inculquer à l'enfant le respect du milieu naturel. »

15 L'UNESCO, pour sa part, a adopté en 1974 une recommandation sur l'éducation pour la compréhension, la coopération, la paix internationales et l'éducation relative aux droits de l'homme et aux libertés fondamentales. L'actualité de la recommandation a été affirmée à la Conférence internationale de l'éducation en octobre 1994. La recommandation contient une section sur la préparation des éducateurs et enseignants dans laquelle on trouve notamment que les États membres devraient faire leur possible pour :

développer chez les éducateurs les motivations de leur action ultérieure : adhésion à l'éthique des droits de l'homme et à l'objectif de changer la société afin de réaliser les droits de l'homme dans les faits, sens de l'unité fondamentale de l'humanité, capacité d'inculquer le sentiment des richesses que la diversité des cultures apporte à chaque personne, groupe ou peuple; offrir un bagage de connaissances interdisciplinaires sur les problèmes mondiaux et les problèmes de la coopération internationale, notamment grâce à un travail relatif à la solution de ces problèmes.

Dans un document d'accompagnement de la recommandation, Brigitte Reich affirme ${ }^{7}$ :

Dans la plupart des pays du monde, la formation traditionnelle des enseignants se concentre sur l'apprentissage des disciplines et beaucoup moins sur le développement de compétences sociales pour faire face aux nouveaux défis qui émergent de changements dans les conditions sociales qui influencent la socialisation des enfants. Parmi ces changements nous pouvons citer les évolutions technologiques, l'influence dominante des mass medias, la dévaluation de valeurs traditionnelles et la recherche de nouvelles orientations et valeurs humaines. S'ils ne reçoivent pas une formation qui leur permette d'affronter ces changements et de réfléchir à de nouvelles conditions et de nouveaux besoins, les enseignants ne seront pas favorables à des initiatives de réformes et ils peuvent même freiner leur progrès.

17 Sa conclusion est qu'une formation aux disciplines devrait être accompagnée d'une formation à l'éducation à la compréhension internationale, ses objectifs, ses valeurs et ses pédagogies.

Bien sûr la mise en action de ces recommandations a ses propres problématiques. Un autre document de l'Unesco en présente deux aspects qui me semblent particulièrement pertinents ${ }^{8}$. Ayant étudié les réponses à un questionnaire envoyé à tous les États membres, le document constate que : «Il y a actuellement une tendance à définir les objectifs éducatifs selon une reconnaissance implicite ou explicite de la tension entre le 
renforcement d'une identité et d'une spécificité culturelle d'un côté et l'ouverture vers l'universel de l'autre ".

Cette problématique devrait être au cœur de toute réflexion sur la formation des enseignants à une dimension internationale. Dans cette réflexion, on est inévitablement confronté à d'autres questions :

Comment promouvoir les cultures des différents groupes représentés dans un pays tout en évitant les nationalismes primaires de certains mouvements ethniques? Comment réconcilier une éducation patriotique/nationale et un apprentissage des valeurs européennes et mondiales? Quelle place accorder à l'éducation concernant les religions? Toutes ces interrogations confirment que l'intériorisation des valeurs et des connaissances qui sont à la base d'une culture de la démocratie et de la paix est un processus lent et complexe qui est dérivé essentiellement d'une pratique et d'une expérience sociale. On peut donc supposer que l'écart entre les changements rapides observés à un niveau institutionnel politique d'un côté et les changements culturels subséquents beaucoup plus lents de l'autre, créeront une période d'incertitude et de conflit. Dans ce contexte la priorité pour l'éducation est d'accélérer l'accès des citoyens aux connaissances et aux valeurs leur permettant de réduire les incertitudes et les coûts sociaux et individuels des conflits.

Voilà précisément tout l'enjeu éducatif d'une dimension internationale. Les enseignants sont aux premières lignes d'une défense de la paix sociale et internationale. Ils ne sont pas les seuls acteurs, mais ils sont indispensables à un projet de création d'un monde de justice et de paix. Aux formateurs des enseignants de les aider à transformer la rhétorique des grandes instances internationales en une réalité dans leurs classes et dans leurs écoles.

\section{NOTES}

1. Ministère de l'éducation nationale, Rapport de l'Inspection générale de l'éducation nationale, Paris, La Documentation française, 1994, p. 542-544.

2. Christian Nique, L'impossible gouvernement des esprits, Paris, Nathan, 1991, p. 151.

3. Jules Ferry, Lettre adressée aux instituteurs (1883), in: François Gaspard et Farhad Khosrokhavar, Le foulard et la République, Paris, La Découverte, 1995, p. 178.

4. L'assemblée parlementaire du Conseil de l'Europe a adopté le 24 janvier 1994 une recommandation sur l'égalité des droits entre femmes et hommes dans laquelle il est recommandé au comité des ministres de dépasser l'ambiguïté de la formule « droits de l'homme » en le remplaçant par celle de « droits de la personne »

5. Traduction de l'auteur.

6. Bronislaw Geremek, L'apprentissage interculturel au service des droits de l'homme, Strasbourg, Conseil de l'Europe, 1991.

7. B. Reich et V. Pivovarov, International Practical Guide on the Implementation of the Recommendation concerning Education for International Understanding, etc., Paris, Unesco (preliminary edition), 1994.

8. Unesco, La situation de l'éducation à la compréhension internationale, document de travail pour la $44^{\mathrm{e}}$ conférence internationale de l'éducation, Genève, 4-8 octobre 1994 (ED/BIE/CONFINTED/44/ INF.2). 


\section{RÉSUMÉS}

La dimension nationale traçait au XIX ${ }^{\mathrm{e}}$ siècle l'horizon de l'éducation civique. Aujourd'hui, la mission des enseignants est de former des citoyens capables de trouver leur place dans un monde de nations de plus en plus interdépendantes et traversé par des mutations de tous ordres. Ces changements rapides et les menaces qu'ils font peser sur les valeurs de la démocratie, appellent une éducation internationale fondée sur une éthique commune des droits de l'homme. Un rappel des recommandations des grandes instances internationales - Conseil de l'Europe, UNESCO, UNICEF.

\section{INDEX}

Index géographique : Europe, France, Angleterre

Mots-clés : Conseil de l'Europe, éducation internationale, formation des enseignants, instruction civique, pluralisme culturel, Unesco

\section{AUTEUR}

\section{HUGH STARKEY}

Expert auprès du Conseil de l'Europe pour les droits de l'homme, Westminster College, Oxford, Grande-Bretagne 